7. Für eine geburtsvorbereitende Akupunktur gibt es keinerlei Evidenz. „Vielleicht gibt es einen Wohlfühleffekt für die Frau, aber die Geburt verläuft nicht schneller oder besser", betonte Schild.

Auch die meisten individuellen Gesundheitsleistungen sind laut Schild nicht evidenzbasiert. Er forderte: „Wir müssen aufklären, ohne Angst zu machen“ und konstatierte: „Mehr ist nicht grundsätzlich besser." Eine besonders überflüssige

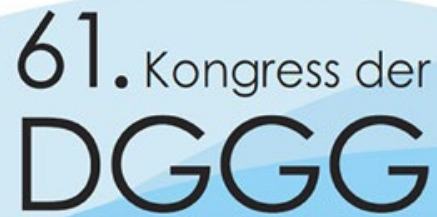

Maßnahme sei, dass schwangere Ärztinnen nicht mehr operieren dürfen. „Das gibt es nur in Deutschland“, erklärte Schild. „Das ist für Frauen, die arbeiten wollen, wirklich nicht förderlich für die Karriere." Er verwies auf das Positionspapier "Operieren in der Schwangerschaft" (OPidS) des Jungen Forums der Deutschen Gesellschaft für Orthopädie und Unfallchirurgie (DGOU) in Zusam- menarbeit mit dem Perspektivforum Junge Chirurgie. Dort gebe es auch entsprechende Merkblätter, wie man das Operieren bei gleichzeitigem Schutz von Schwangerer und Fötus möglich machen kann [http://www.opids.de/fileadmin/ user_upload/OPIDS/Dokumente/Tools/ Positionspapier_OPidS_FINAL.pdf].

Friederike Klein, München

\section{Geburtseinleitung nach Evidenz oder Label?}

\section{Zur Geburtseinleitung wird vielfach orales Misoprostol eingesetzt, das für diese Indikation nicht zugelassen ist. Andere Optionen sind Dinopreston oder Ballonkatheter und das seit Kurzem auf dem Markt erhältliche Misoprostol- Vaginalinsert, über das beim Kongress erheblich debattiert wurde.}

W ie Privatdozent Sven Kehl, Frauenklinik der Universität Erlangen, erklärte, wird jede vierte bis fünfte Geburt in Deutschland eingeleitet [Hehl S et al. DGGG 2016, FV050]. Evidenzbasierte Verfahren sind dabei die orale Gabe von Misoprostol (MO, Cyrtotec ${ }^{\varpi}$ ) oder der Ballonkatheter. Nach einer multizentrischen Kohortenstudie war die sequenzielle Kombination beider Methoden mit signifikant weniger Kaiserschnitten als eine Einleitung mit oralem Misprostol alleine assoziiert (17,3 vs. $26,1 \%)$. Das galt unabhängig von der $\mathrm{Pa}$ rität (24,2 vs. $37,2 \%$ bei Erstgebärenden; 7 vs. 9,1\% bei Mehrgebärenden). Auch das Einleitungs-Geburtsintervall war unabhängig von der Parität bei sequenzieller Kombination - zuerst Katheter, dann Misoprostol - um im Mittel 342 Minuten kürzer, die Rate vaginaler Geburten innerhalb von 24 Stunden $(64,7$ vs. $51,9 \%)$ und von 48 Stunden (95,8 vs. $87,4 \%)$ signifikant höher. Kehl habe gute Erfahrungen damit gemacht, den Ballonkatheter abends einzulegen und über Nacht liegen zu lassen. Wenn nach dem Ziehen am Morgen die Geburt nicht einsetze, könne die orale Misoprostol-Gabe erfolgen. Der Ballonkatheter wird in Deutschland zur Geburtseinleitung allerdings bislang relativ selten verwendet. Nach einer deutschlandweiten Befragung im Mai und Juli 2013, an der sich
$69 \%$ der 738 angeschriebenen geburtshilflichen Kliniken beteiligten, setzen mehr als zwei Drittel bei reifer Zervix nie einen Ballonkatheter ein. In Norwegen geben dagegen $80 \%$ aller Kliniken an, den Ballonkatheter zu verwenden [Voigt $\mathrm{F}$ et al. DGGG 2016, P554]. Am häufigsten setzten die deutschen Geburtshelfer 2013 orales Misoprostol ein - trotz der nicht vorhandenen Zulassung. Inzwischen wurde ein Vaginalinsert mit Misoprostol (Misodel ${ }^{\circledR}, \mathrm{MVI}$ ) zugelassen. Ergebnisse einer deutschen Kohortenstudie zeigen eine vergleichbare Einleitungsdauer wie bei oralem Misoprostol (823 vs. 847 Minuten für MO) und eine Überlegenheit gegenüber einem Vaginalinsert mit dem Prostaglandin E2 Dinoproston (DVI, Propess ${ }^{\oplus}, 1.226 \mathrm{Mi}$ nuten). Eine vaginale Entbindung innerhalb von 24 Stunden erfolgte mit MVI bei $85,5 \%$ der Frauen, bei MO bei $87,5 \%$ und bei DVI bei $64 \%$. Oxytocin zur Wehenaugmentation musste in der MVIGruppe nur bei 8,2\% der Frauen eingesetzt werden, bei MO war das bei $24,3 \%$ und bei DVI bei $36 \%$ der Frauen erforderlich. Allerdings waren uterine Polysystolien bei dem MVI mit $20 \%$ signifikant häufiger als bei $\mathrm{MO}(1,4 \%)$ und DVI (4,7\%). Das korrespondierte mit einer erhöhten Rate von CTG-Komplikationen $(51,8,27$ und $28,1 \%$ in den drei Gruppen), aber nicht mit einer signifi- kant häufigeren Notwendigkeit sekundärer Kaiserschnitte (24,5\% bei MVI-, $18,9 \%$ bei MO- und $26,6 \%$ bei DVI-Einsatz) oder einer erhöhten perinatalen Mortalität, wie Dr. Sven Seeger von der Klinik für Frauenheilkunde und Geburtshilfe in Halle betonte.

Vorangegangene Studien hatten auf eine höhere Sektiorate bei dem MVI hingewiesen, was Prof. Wolfgang Henrich, Berlin, in der Diskussion auf eine zu hohe Misoprostoldosis im Präparat zurückführte. Er sagte: „Ich habe den Eindruck, es ist wirklich nur der medikolegale Druck, der verhindert, dass das orale Misoprostol das Einleitungspräparat Nummer 1 ist." Dabei gebe es in anderen Fällen durchaus die Rechtsprechung, dass ein nicht zugelassenes Präparat, das wissenschaftlich belegbar besser oder nebenwirkungsärmer ist als ein zugelassenes, auch eingesetzt werden müsse. Sein Standpunkt: „Wenn wir es nicht einsetzen, ist das ein Behandlungsfehler!“

In der Diskussion forderte die DGGG ein positives Statement zur Geburtseinleitung mit Misoprostol mit klaren Indikationen und Kontraindikationen, wie es die schweizerische Fachgesellschaft getan habe [SGGG. Expertenbrief Nr. 38 Misoprostol zur Geburtseinleitung vom 1. Juni 2011]. Prof. Maritta Kühnert, Marburg, warb um Verständnis dafür, dass die DGGG bei fehlender Zulassung nicht mehr tun könne, als auf die individuelle Therapiefreiheit des Arztes und die Notwendigkeit der Information der Patientin über das Off-LabelProblem und die entsprechende Aufklärung über eventuelle Konsequenzen hinzuweisen.

Friederike Klein, München 